\title{
Stage III Pancreatic Neuroendocrine Tumor AJCC v8
}

National Cancer Institute

\section{Source}

National Cancer Institute. Stage I/I Pancreatic Neuroendocrine Tumor A/CC v8. NCI

Thesaurus. Code C135563.

Stage III includes: (T4, N0, M0); (Any T, N1, M0). T4: T umor invading adjacent organs (stomach, spleen, colon, adrenal gland) or the wall of large vessels (celiac axis or the superior mesenteric artery). N0: No regional lymph node involvement. N1: Regional lymph node involvement. M0: No distant metastasis. (AJCC 8th ed.) 